\title{
Characterization of epitope specificity of Proteus penneri 7 lipopolysaccharide core region
}

\author{
Agata Palusiak ${ }^{\varpi}$ and Zygmunt Sidorczyk \\ Department of General Microbiology, Institute of Microbiology, Biotechnology and Immunology, Faculty of Biology and Environmental Protec- \\ tion, University of Łódź, Łódź, Poland
}

To extend the knowledge on the fragments of Proteus penneri lipopolysaccharide core regions, which determine the cross-reactions with specific antibodies, serological studies were performed by use of $P$. penneri 7 core-specific antiserum and Proteus sp. lipopolysaccharides. Different reactivity of the tested antiserum with three groups of antigens suggested differences in their core regions' epitope specificity. Comparing the results of the serological investigations with the previously determined structures of the core regions of the tested $P$. penneri lipopolysaccharides allowed distinguishing two potential tri- and tetrasaccharide epitopes and a third fragment which could not be determined precisely.

Keywords: Proteus penneri, lipopolysaccharide, epitope, core region

Received: 14 April, 2010; revised: 07 October, 2010; accepted: 03 November, 2010; available on-line: 09 November, 2010

\section{INTRODUCTION}

The lipopolysaccharide (LPS) core region of Proteus penneri strains is characterized by higher structural variability than that observed in other representatives of Enterobacteriaceae. Thus, twelve different structures of the distal part of P. penneri LPS core region and three structural variants of its proximal part have been determined, whereas only five structural types of the whole core region of Escherichia coli LPSs and two such types of Salmonella enterica LPSs have been identified (Vinogradov et al., 2002b; Holst, 2007). It seemed interesting whether such high structural heterogeneity of $P$. penneri core region also refers to the number of its fragments responsible for the cross-reactions with specific antibodies. Up to date only a few epitopes have been determined in the $P$. penneri LPS core region (Palusiak et al., 2008). Other published data concerning similar epitopes referred to other species of genus Proteus: P. mirabilis and P. vulgaris, and the epitopes were determined by use of antisera containing both O-polysaccharide- and core region-specific antibodies (Sidorczyk et al., 2002b; Kołodziejska et al., 2006). Using in this work an antiserum which contained only the latter type of antibodies enabled a more precise determination of exclusively the core region epitopes.

\section{MATERIALS AND METHODS}

Bacterial strains, lipopolysaccharides and antiserum. In all, 41 Proteus sp. strains were used. P. penneri 7 (O61) was a clinical isolate from the faeces of a patient from Missouri (USA). This strain and 26 others (includ- ing: P. penneri 8 (O67), 13 (R form), 14 (O59), 15 (O52) and $34(\mathrm{O} 65)$ ) were kindly provided by Professor D. J. Brenner, Center for Diseases Control and Prevention in Atlanta (GA, USA). Three $P$. penneri strains were isolated from the urine of women with bacteriuria in a hospital in Lódź (Poland). One P. penneri and one P. mirabilis strain came from the collection of the Department of General Microbiology, University of Łódź (Poland). Five strains, including P. penneri 104 (O61), were from Dr. B. Holmes, Central Public Health Laboratory in London (UK) and one strain P. penneri 133 (O61) from Dr. G. Giammanco, Institute of Hygiene and Prevention Medicine in Catania (Italy). The remaining three strains were kindly provided by Dr. E. Falsen, Department of Clinical Bacteriology, Göteborg (Sweden) and Dr. B. Senior, Department of Medical Microbiology in Dundee (UK).

The cultivation of bacteria, isolation and saponification of the LPSs, the immunization procedure and obtaining of antiserum were carried out as was described in detail earlier (Westphal \& Jann, 1965; Sidorczyk et al., 2002b).

Serological assays. Non-absorbed P. penneri 7 antiserum was checked with a set of forty-one Proteus sp. LPSs in an enzymatic immunosorbent assay (ELISA). Homologous and cross-reactive LPSs were tested with this antiserum by Western blot technique. In this procedure LPS samples were prepared similarly to the method described elsewhere (Milnerowicz \& Bizoń, 2010). The antiserum was absorbed with homologous and respective cross-reactive LPSs and checked with them in the hemagglutination procedure. The first two methods and the absorption procedure were carried out as described in detail elsewhere (Sidorczyk et al., 2002b; Palusiak et al., 2008). Antibody titre determination by ELISA was repeated three times for each system.

Hemagglutination procedure. Alkali-treated LPSs were used for sensitizing sheep red blood cells (SRBC) for $30 \mathrm{~min}$ at $37^{\circ} \mathrm{C}(200 \mu \mathrm{g} \mathrm{LPS} / 0.2 \mathrm{ml} \mathrm{SRBC})$. Such sensitized erythrocytes were washed twice with phosphate-buffered saline (PBS) pH $7.2\left(15 \mathrm{mM} \mathrm{NaH} \mathrm{PO}_{4}\right.$, $150 \mathrm{mM} \mathrm{NaCl}$ ) and suspended in the same buffer containing $0.1 \%$ bovine serum albumin (BSA) to give $0.1 \%$ suspension. To serial twofold dilutions of antiserum, 50 $\mu \mathrm{l}$ PBS buffer with $0.1 \%$ BSA and $50 \mu \mathrm{l}$ suspension of the sensitized SRBC were added to each well of the plate

e-mail: agatapal@biol.uni.lodz.pl

Abbreviations: Arap4N, 4-amino-4-deoxy-L-arabinopyranose; ELISA, enzymatic immunosorbent assay; GalA, galacturonic acid; GalN galactosamine; GalNA, amides of GalA with aliphatic polyamines; Glc, glucose; GlcN, glucosamine; GlcNGly, 2-deoxy-2-glycylaminoD-glucose; Hep, L-glycero-D-manno-heptose; DD-Hep, D-glycero-Dmanno-heptose; Kdo, 3-deoxy-D-manno-oct-2-ulosonic acid; LPS, lipopolysaccharide; PEtn, 2-aminoethyl phosphate. 
(incubation for $1 \mathrm{~h}$ at $4{ }^{\circ} \mathrm{C}$ and for $16 \mathrm{~h}$ at $37^{\circ} \mathrm{C}$ ). The titre was established as the highest dilution of antiserum resulting in 50\% hemagglutination (Schmidt et al., 1970).

\section{RESULTS}

This work presents serological characterization of the $P$. penneri 7 LPS core region. First, rabbit polyclonal antiserum against heat-killed $P$. penneri 7 was absorbed with alkali-treated LPS from P. penneri strain 104, whose LPS has an O-polysaccharide chain serologically identical to that of P. penneri 7 LPS and a different core region (unpublished). That procedure gave an antiserum containing antibodies against the core region only. The elimination of the O-polysaccharide-specific antibodies from the antiserum was confirmed by Western blotting after sodium dodecyl sulfate polyacrylamide gel electrophoresis (SDS/ PAGE) of LPS preparations from the strains tested (Fig. 1). As expected, the tested antiserum did not react with the high-molecular mass O-polysaccharide containing LPS species of $P$. penneri 104 and 7 strains, which confirmed the efficiency of the absorption procedure. This serum will be further referred to as core-specific antiserum.

The reactivity of the tested antiserum was checked by ELISA with a set of forty-one Proteus sp. LPSs selected on the basis of the results of previous serological studies conducted in the Department of General Microbiology (Zych et al., 2000; 2005; Sidorczyk et al., 2002a; Kondakova et al., 2003; Drzewiecka et al., 2004). The coreantiserum reacted with seven LPSs. The strongest and identical reactions were observed with $P$. penneri 7,14 and 15 LPSs (reciprocal titre: 256000), and a less strong one with $P$. penneri 13 (reciprocal titre: 16000). LPSs of P. penneri 8, 34 and 133 bound the antibodies with the lowest but identical strength (reciprocal titre: 8000).

In the Western blot, whose main aim was to show which region of LPSs was involved in the observed reactions, the tested antiserum clearly recognized the lowmolecular-mass LPS species restricted to the core-lipid A moiety from the homologous and six cross-reactive P. penneri strains (Fig. 1). Similarly to the results obtained in ELISA, the strongest reactions were observed for P. penneri 7,14 and 15 LPSs.

In the next step of the investigation, the core-antiserum was first absorbed with the applied seven LPSs and then tested with these LPS preparations in the hemagglutination procedure. Each time the antiserum was absorbed with the LPS of one $P$. penneri strain. The absorption of the tested antiserum with any of the $P$. pen-

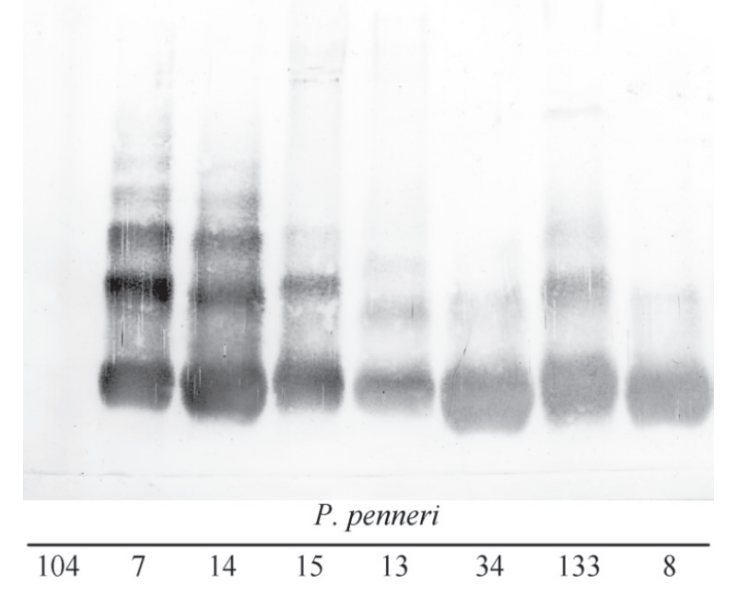

Figure 1. Western blot of $P$. penneri LPSs with core-specific antiserum against $P$. penneri 7 .

The antiserum was diluted 1:100 in dot-blot-10\% skimmed milk buffer. The reactions were developed with alkaline phosphateconjugated goat anti-rabbit antibodies diluted 1:2000. Alkaline Phosphatase Conjugate Substrate Kit (Bio-Rad) was used.

neri 7,14 or 15 LPSs completely abolished its reactivity with all the antigens tested, which suggests that the core regions of these three LPSs were serologically identical (Table 1). The absorption of antiserum with the second group of identically reactive antigens, $P$. penneri 8,34 and 133 LPSs, caused a complete elimination of antibodies to these antigens and to P. penneri 13 LPS, and decreased the antiserum titres with $P$. penneri 7,14 and 15 LPSs. This result indicates some differences between epitopes located in LPS core regions of P. penneri 7, 14, 15, 13 and those of the second group: P. penneri 8, 34 and 133.

After the absorption of the core-antiserum with $P$. penneri 13 LPS, the reactivity with $P$. penneri 7,14 or 15 was markedly decreased and only slightly decreased in the case of the other three antigens P. penneri 8,34 and 133. The antibodies removed by this absorption from the antiserum probably bound to another common fragment located in the core region of all the mentioned LPS. Accordingly, immunoglobulins that remained in the antiserum absorbed in this way recognized core region fragments which differ $P$. penneri 13 LPS and all the remaining LPSs tested.

Table 1. Hemagglutination of alkali-treated $P$. penneri LPSs with absorbed core-specific antiserum against $P$. penneri $7^{\mathrm{a}, \mathrm{b}}$ aNon-absorbed core-specific antiserum was used as control; bData for homologous LPS are italicized.

\begin{tabular}{|c|c|c|c|c|c|c|c|}
\hline \multirow{2}{*}{$\begin{array}{l}\text { Alkali-treated LPS used } \\
\text { for absorption }\end{array}$} & \multicolumn{7}{|c|}{ Reciprocal titer of absorbed core-specific antiserum for alkali-treated LPS from $P$. penneri strains } \\
\hline & 7 & 14 & 15 & 8 & 34 & 133 & 13 \\
\hline Control & 51200 & 51200 & 51200 & 3200 & 3200 & 3200 & 1600 \\
\hline P. penneri 7 & $<100$ & $<100$ & $<100$ & $<100$ & $<100$ & $<100$ & $<100$ \\
\hline P. penneri 14 & $<100$ & $<100$ & $<100$ & $<100$ & $<100$ & $<100$ & $<100$ \\
\hline P. penneri 15 & $<100$ & $<100$ & $<100$ & $<100$ & $<100$ & $<100$ & $<100$ \\
\hline P. penneri 8 & 12800 & 12800 & 12800 & $<100$ & $<100$ & $<100$ & $<100$ \\
\hline P. penneri 34 & 12800 & 12800 & 12800 & $<100$ & $<100$ & $<100$ & $<100$ \\
\hline P. penneri 133 & 12800 & 12800 & 12800 & $<100$ & $<100$ & $<100$ & $<100$ \\
\hline P. penneri 13 & 1600 & 1600 & 1600 & 800 & 800 & 800 & $<100$ \\
\hline
\end{tabular}


a. P. penneri $7\left(\mathrm{R}^{3}=\mathrm{H}\right)($ Vinogradov et al., 2002a)

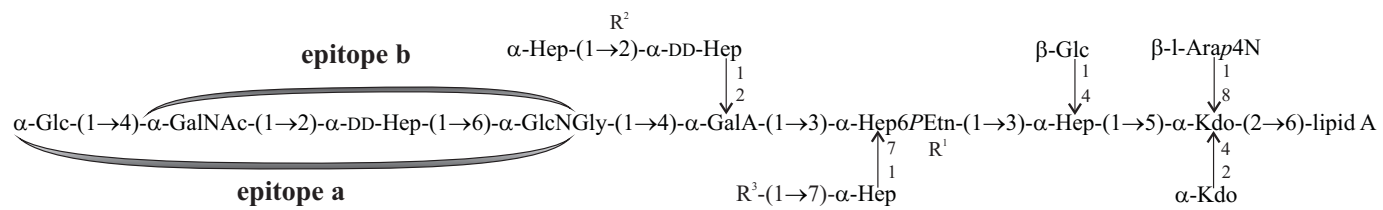

b. P. penneri $14\left(\mathrm{R}^{1}=P\right.$ Etn or $\mathrm{H} ; \mathrm{R}^{3}=\mathrm{H}$ or $\left.\beta-G a l N A\right)$ (Vinogradov et al., 2002a)

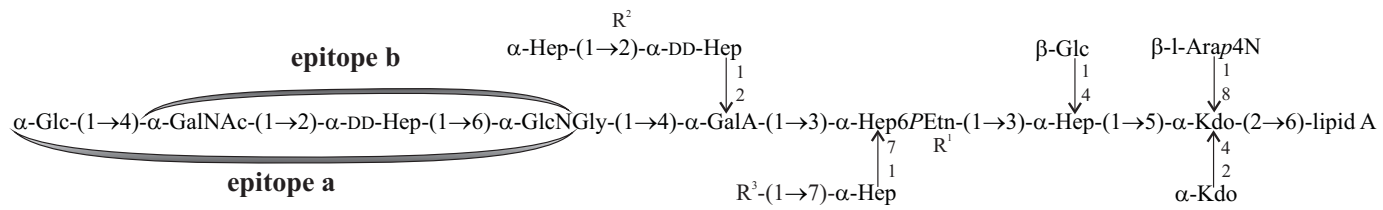

c. P. penneri $15\left(\mathrm{R}^{3}=\mathrm{H}\right)$ (Vinogradov et al., 2002a)

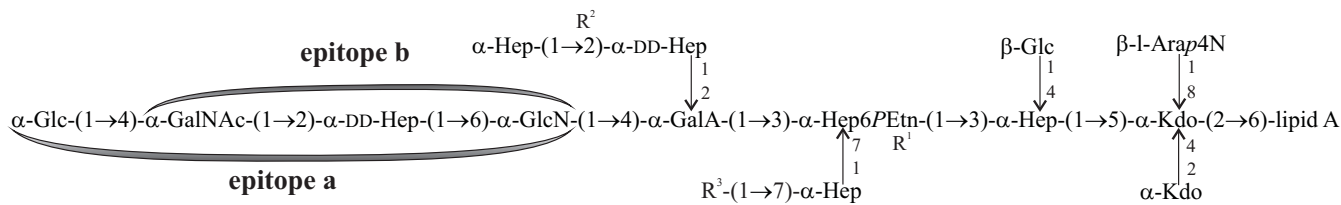

d. P. penneri $8\left(\mathrm{R}^{3}=\mathrm{H}\right)$ (Vinogradov et al., 2002a)

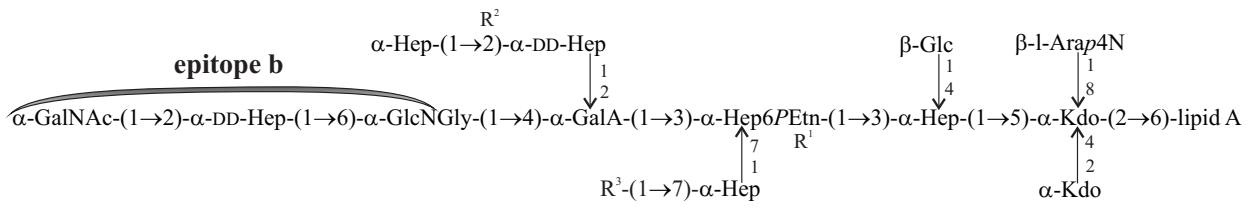

e. P. penneri $13\left(\mathrm{R}^{1}=\right.$ PEtn or $\mathrm{H} ; \mathrm{R}^{3}=\mathrm{H}$ or $\beta$-GalAN) (Vinogradov \& Sidorczyk, 2002)

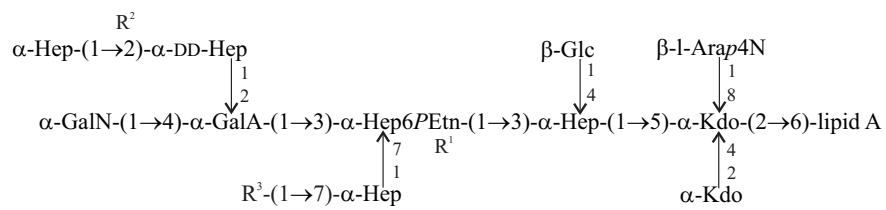

Figure 2. Structures of core-lipid A region of $P$. penneri LPSs

Common epitopes are indicated by arcs. Arap4N, 4-amino-4-deoxy-L-arabinopyranose; GalA, galacturonic acid; GalN, galactosamine; GalNA, amides of GalA with aliphatic polyamines; Glc, glucose; GlcN, glucosamine; GlcNGly, 2-deoxy-2-glycylamino-D-glucose; Hep, L-glyceroD-manno-heptose; DD-Hep, D-glycero-D-manno-heptose; Kdo, 3-deoxy-D-manno-oct-2-ulosonic acid; PEtn, 2-aminoethyl phosphate.

\section{DISCUSSION}

The outcome of the serological studies reported here can be referred to the previously determined chemical structures of the core regions of $P$. penneri $7,14,15,8$ and 13 LPSs (Fig. 2a, b, c, d, e) (Vinogradov \& Sidorczyk, 2002; Vinogradov et al., 2002a). Our analyses, especially the results of hemagglutination with antisera after their absorption with an appropriate LPS sample (Table 1), allowed suggesting that the cross-reactions of the tested antiserum were determined by three common fragments located in the core regions of homologous and respective cross-reactive LPSs. The first epitope, marked in Fig. 2 as epitope a, seems to be associated with the following tetrasaccharide fragment: $\alpha$-Glc-
$(1 \rightarrow 4)-\alpha$-GalNAc- $(1 \rightarrow 2)-\alpha$-DDHep- $(1 \rightarrow 0)-\alpha-G l c N$ found in the distal part of $P$. penneri 7,14 and 15 LPS core regions (Fig. 2a, b, c). Additionally, P. penneri 7 and 14 LPS core regions have a glycine residue attached to $\alpha-G l c N$ of epitope a. The lack of reaction of core-antiserum absorbed with $P$. penneri 15 LPS with LPSs which possess this amino acid in their core regions $(P$. penneri 7 and 14) suggests that the glycine residue does not play a role in the epitope specificity.

The second fragment referred to as epitope $b$, $\alpha$-GalNAc- $(1 \rightarrow 2)-\alpha$-DDHep- $(1 \rightarrow 0)-\alpha$-GlcN, $\quad$ occupying distal part of $P$. penneri $7,14,15$ and 8 LPS core regions might account for the respective cross-reactions observed in this work (Fig. 2a, b, c, d). This epitope might also be located in the LPS core regions of P. penneri 34 and 133 , 
whose core oligosaccharide structures remain unknown. This suggestion could be made only on the grounds of the identical serological reactivity of the tested antiserum with $P$. penneri 34, 133 and 8 LPSs observed in this work. It is worth mentioning that these data are also confirmed by the results of immunochemical studies obtained with $P$. penneri 8 anti-conjugate and anti-core sera, which also indicated the presence of epitope $\mathrm{b}$ in the $P$. penneri 8 , 34 and 133 LPS core regions (Palusiak et al., 2008). This fragment differs from epitope a by the lack of the terminal glucose residue. The higher antiserum titres with LPSs having the terminal glucose residue in their core regions ( $P$. penneri 7,14 and 15$)$ compared with those obtained with LPSs which do not have it $(P$. penneri 8 , and probably $P$. penneri 34 and 133) showed that this component may largely contribute to the epitope specificity of the homologous and P. penneri 14 and 15 antigens. The reaction of the tested antiserum after its absorption with $P$. penneri 13 LPS indicated the presence of a third epitope located in the core region of $P$. penneri 7 and the remaining LPSs. A comparison of the core region structures of P. penneri 7 and 13 LPSs showed that they share most components in the inner part of the core oligosaccharide and their distal parts of the core region are completely different (Fig. 2a, e). This fact indicates that this third epitope is associated with a fragment of the inner part of the core regions of all the investigated LPSs. However, the great similarity of the mentioned part of all LPSs core regions makes it impossible to identify the fragment acting as the third epitope. The most likely option is that $\alpha$-Hep- $(1 \rightarrow 2)-\alpha$-DD-Hep could play a role of such an epitope. That residue occupied the most distal fragment of the inner part of the core oligosaccharide of all LPSs studied (Fig. 2a, b, c, d, e) and that is why it may have been quite easily recognized by the specific antibodies. Apart from these assumptions, the third epitope located in the core regions of $P$. penneri 7 and other investigated LPSs remains unknown.

Gathering the information on the presence of common epitopes in $P$. penneri LPS core regions should be helpful in finding $\mathrm{R}$ antigens of LPS, necessary for preparing a vaccine protecting not only against one Proteus sp. strain but also effective with other strains for which it will be possible to obtain protective immunoglobulins of broad specificity.

\section{Acknowledgements}

This work was supported by the Ministry of Science and Higher Education (Poland), grant No. N 401001 31/0009.

\section{REFERENCES}

Drzewiecka D, Zych K, Sidorczyk Z (2004) Characterization and serological classification of a collection of Proteus penneri clinical strains. Arch Immunol Ther Exp 52: 121-128.

Holst O (2007) The structures of core regions from enterobacterial lipopolysaccharides - an update. FEMS Microbiol Lett 271: 3-11.

Kołodziejska K, Perepelov AV, Zabłotni A, Drzewiecka D, Senchenkova SN, Zych K, Shashkov AS, Knirel YA, Sidorczyk Z (2006) Structure of the glycerol phosphate-containing O-polysaccharides and serological studies of the lipopolysaccharides of Proteus mirabilis CCUG 10704 (OE) and Proteus vulgaris TG 103 classified into a new Proteus serogroup, O54. FEMS Immunol Med Microbiol 47: 267-274.

Kondakova AN, Zych K, Senchenkova SN, Bartodziejska B, Shashkov AS, Knirel YA, Różalski AA, Sidorczyk Z (2003) Structure of the O-polysaccharide of Proteus penneri 28 and Proteus vulgaris O31 and classification of P. penneri 26 and 28 in Proteus serogroup O31. FEMS Immunol Med Microbiol 39: 87-93.

Milnerowicz H, Bizoń A (2010) Determination of metallothionein in biological fluids using enzyme-linked immunoassay with commercial antibody. Acta Biochim Polon 57: 99-104.

Palusiak A, Dzieciątkowska M, Sidorczyk Z (2008) Application of two different kinds of sera against the Proteus penneri lipopolysaccharide core region in serach of epitopes determining cross-reactions with antibodies. Arch Immunol Ther Exp 56: 135-140.

Schmidt G, Fromme J, Mayer H (1970) Immunochemical studies on core lipopolysaccharides of Enterobacteriaceae of different genera. Eur J Biochem 14: 357-366.

Sidorczyk Z, Toukach FV, Zych K, Arbatsky NP, Drzewiecka D, Ziółkowski A, Shashkov AS, Knirel YA (2002a) Structural and serological characterization of the lipopolysaccharide from Proteus penneri 20 and classification of the cross-reacting Proteus penneri strains 10, 16, 18, 20, 32 and 45 in Proteus serogroup O17. Arch Immunol Ther Exp 50: 345-350.

Sidorczyk Z, Zych K, Toukach FV, Arbatsky NP, Zabłotni A, Shashkov AS, Knirel YA (2002b) Structure of the O-polysaccharide and classification of Proteus mirabilis strain G1 in Proteus serogroup O3. Eur J Biochem 269: 1406-1412.

Vinogradov E, Sidorczyk Z (2002) The structure of the carbohydrate backbone of the rough type lipopolysaccharides from Proteus penneri strains 12, 13, 37 and 44. Carbohydr Res 337: 835-840.

Vinogradov E, Sidorczyk Z, Knirel YA (2002a) Structure of the core part of the lipopolysaccharides from Proteus penneri strains 7, 8, 14, 15, and 21. Carbobydr Res 337: 643-649.

Vinogradov E, Sidorczyk Z, Knirel YA (2002b) Structure of the lipopolysaccharide core region of the bacteria of the genus. Proteus. Aust J Chem 55: 61-67.

Westphal O, Jann K (1965) Bacterial lipopolysaccharides. Extraction with phenol/water and further application of the procedure. Methods Carbobydr Chem 5: 83-91.

Zych K, Kocharova NA, Kowalczyk M, Toukach FV, Kamińska D, Shashkov AS, Knirel YA, Sidorczyk Z (2000) Structure of the Ospecific polysaccharide of Proteus penneri 71 and classification of cross-reactive $P$. penneri strains to a new proposed serogroup O64. Eur J Biochem 267: 808-814.

Zych K, Perepelov AV, Baranowska A, Zabłotni A, Knirel YA, Sidorczyk Z (2005) Structure and serological studies of the O-polysaccharide of Proteus penneri 75. Epitopes and subgroups of Proteus serogroup O73. FEMS Immunol Med Microbiol 43: 141-148. 